\title{
The General Stationary Gravitational Vacuum Field of Cylindrical Symmetry
}

\author{
ECKART FREHLAND \\ Physikalisches Institut der Universität Freiburg, Abteilung Theoretische Physik, \\ Freiburg i. Br.
}

Received June 14, 1971

Abstract. The general stationary vacuum gravitational field of cylindrical symmetry as recently found by Davies and Caplan is even static. The possible Petrov types of the Riemann tensor are $I, D$ or $O$. In spacelike infinity the spacetime becomes necessarily flat.

\section{Introduction}

Levy and Robinson [1] have argued that for axisymmetric stationary systems and modulo the vacuum field equations

$$
R_{\mu \nu}=0
$$

there exists a canonical (cylindrical) coordinate system in which the line element takes the form

$$
\begin{aligned}
d s^{2}=e^{2 u}(d t+a d \varphi)^{2} & -e^{2(k-u)}\left(d r^{2}+d z^{2}\right) \\
& -r^{2} e^{-2 u} d \varphi^{2}
\end{aligned}
$$

$a=0$ corresponds to Weyl's canonical coordinates for the static case. If $u, k, a$ are functions of $r$ only, the line element represents the vacuum gravitational field within or outside an infinite, axially symmetric rotating cylindrical mass distribution and the field Eq. (1.1) reduce to

$$
\begin{gathered}
\frac{d^{2} u}{d r^{2}}+\frac{1}{r} \frac{d u}{d r}+\frac{1}{2 r^{2}} e^{4 u}\left(\frac{d a}{d r}\right)^{2}=0 \\
\frac{d^{2} a}{d r^{2}}-\frac{1}{r} \frac{d a}{d r}+4 \frac{d a}{d r} \frac{d u}{d r}=0 \\
\frac{2}{r} \frac{d k}{d r}-2\left(\frac{d u}{d r}\right)^{2}+\frac{1}{2 r^{2}} e^{4 u}\left(\frac{d a}{d r}\right)^{2}=0 .
\end{gathered}
$$

Recently Davies and Caplan [2] have found the general solution of (1.3), from which they deduced, that under the condition $u, a, k$ to be finite at $r=0$ the interior of the rotating cylinder is flat. 
In this paper we shall investigate the Davies-Caplan interior and exterior solution with the following results: it can always be reduced to the static case, i.e. $a=0$. By transformation into normal coordinates with respect to the radial (invariant) distance $R$ we find two special solutions, which as limiting cases are not included in the canonical form (1.2) of the metric.

We show that the general canonical solution is nothing else than the well-known Kasner solution [3]. Therefore the general time-independent vacuum line element, which is considered to be generated by a stationary (rotating) cylindrical mass distribution, is static inside and outside the cylinder, i.e. admits a hypersurface orthogonal timelike Killing field. Possible Petrov types of the Riemann tensor $R^{\alpha \beta}{ }_{\gamma \delta}$ are $I, D$ and $O$. In spacelike infinity $R^{\alpha \beta}{ }_{\gamma \delta}$ vanishes, i.e. the spacetime becomes flat.

\section{The Davies-Caplan Solution}

The general solution of the canonical vacuum Eq. (1.3) is according to Davies and Caplan

$$
\begin{aligned}
& u=\frac{1}{4} \log \left[\frac{\left(1-B \omega^{2 A} r^{2 A}\right)^{2}}{A^{2} B(\omega r)^{2 A-2}}\right] \\
& a=\frac{A}{\omega\left(1-B \omega^{2 A} r^{2 A}\right)} \\
& k=\log \left[D(\omega r)^{\frac{1}{4}(A-1)^{2}}\left(1-B \omega^{2 A} r^{2 A}\right)^{\frac{3}{2}}\right] .
\end{aligned}
$$

Hence, restricting to real values of the metric, the line element is

$$
\begin{aligned}
d s^{2}= & \frac{1-B|\omega|^{2 A} r^{2 A}}{\bar{A}|B|^{\frac{1}{2}}|\omega|^{A-1} r^{A-1}}\left\{d t+\left[\frac{A}{\omega\left(1-B|\omega|^{2 A} r^{2 A}\right)}+C\right] d \varphi\right\}^{2} \\
& -|D|^{2}|A||B|^{\frac{1}{2}}(|\omega| r)^{\frac{1}{2}\left(A^{2}-1\right)}\left(d r^{2}+d z^{2}\right) \\
& -\left(\frac{r^{2} \bar{A}|B|^{\frac{1}{2}}|\omega|^{A-1} r^{A-1}}{1-B|\omega|^{2 A} r^{2 A}}\right) d \varphi^{2}
\end{aligned}
$$

where $|\bar{A}|=|A|$, and the sign of $\bar{A}$ is chosen so that $g_{44}>0$.

In some respect (2.2) resembles the flat-space line element in rotating coordinates. Therefore we try to simplify its form by a linear $\varphi, t$ transformation

$$
\begin{gathered}
t=\alpha \tau+\gamma \phi \\
\varphi=\beta \tau+\delta \phi
\end{gathered}
$$


Indeed, if we set

$$
\alpha=-C \beta, \gamma=-\delta\left(C+\frac{A}{\omega}\right)
$$

we get with a suitable choice of $\alpha, \gamma$ the simplified canonical form

$$
d s^{2}=r^{1-A} d \tau^{2}-r^{\frac{1}{2}\left(A^{2}-1\right)}\left(d r^{2}+d z^{2}\right)-r^{2} r^{A-1} d \phi^{2} .
$$

Hence there is no loss in generality in assuming $a=0$ !

\section{Comparison with the Kasner Solution}

For further discussion it is advantageous to go over to normal coordinates with respect to radial distance $R$ by the transformation

$$
\begin{aligned}
& r=\left(\frac{A^{2}+3}{4}\right) R^{\left(\frac{4}{A^{2}+3}\right)} \\
& \tau=\left(\frac{A^{2}+3}{4}\right)^{\frac{1}{2}(A-1)} \cdot T, \phi=\left(\frac{A^{2}+3}{4}\right)^{-\frac{1}{2}(A+1)} \Phi \\
& z=\left(\frac{A^{2}+3}{4}\right)^{\frac{1}{4}\left(1-A^{2}\right)} \cdot Z .
\end{aligned}
$$

Then the line element is

$$
\begin{aligned}
d s^{2}=- & \left(d R^{2}+R^{4\left(\frac{A+1}{A^{2}+3}\right)} d \Phi^{2}+R^{2\left(\frac{A^{2}-1}{A^{2}+3}\right)} d Z^{2}\right) \\
& +R^{4\left(\frac{1-A}{A^{2}+3}\right)} d T^{2}
\end{aligned}
$$

We shall see below (in Chapter 4.) that (3.2) is solution to the vacuum equations for all real values of $A$, included the limiting cases $A= \pm \infty$, which have to be excluded in the canonical solutions $(2.2),(2.5)$ respectively!

(3.2) is just the general solution found by Kasner ([3] or [4]):

$$
\begin{gathered}
d s^{2}=\mp d x_{1}^{2}-x_{1}^{2 P_{1}} d x_{2}^{2}-x_{1}^{2 P_{2}} d x_{3}^{2} \pm x_{1}^{2 P_{3}} d x_{4}^{2} \\
P_{1}+P_{2}+P_{3}=1, P_{1}^{2}+P_{2}^{2}+P_{3}^{2}=1
\end{gathered}
$$

if we set $x_{1}=R, x_{2}=\Phi, x_{3}=Z, x_{4}=T$. The field is static, i.e. it admits a timelike hypersurface orthogonal Killing vector field

$$
\xi^{\mu}=\delta^{4 \mu}
$$




\section{Properties of the Rieman Tensor}

With (3.2) the Riemann tensor $R^{\alpha \beta}{ }_{\gamma \delta}$ can easily be calculated. The non-vanishing components are in normal coordinates $R, \phi, Z, T$ :

$$
\begin{aligned}
& R^{24}{ }_{42}=R^{31}{ }_{13}=-\frac{4\left(1-A^{2}\right)}{\left(A^{2}+3\right)^{2}} \frac{1}{R^{2}} \\
& R^{34}{ }_{43}=R^{12}{ }_{21}=+\frac{2\left(1-A^{2}\right)(1-A)}{\left(A^{2}+3\right)^{2}} \frac{1}{R^{2}} \\
& R^{32}{ }_{23}=R^{14}{ }_{41}=+\frac{2\left(1-A^{2}\right)(1+A)}{\left(A^{2}+3\right)^{2}} \frac{1}{R^{2}} .
\end{aligned}
$$

Indeed the vacuum equations $R_{\mu \nu}=0$ are fulfilled for all (real) values of $A$, included $A= \pm \infty$.

All components of $R^{\alpha \beta}{ }_{\gamma \delta}$ are proportional to $\frac{1}{R^{2}}$. Hence in the spacelike infinity $(R \rightarrow \infty) R^{\alpha \beta}{ }_{\gamma \delta}$ vanishes and the spacetime becomes flat.

From the diagonal $R^{\alpha \beta}{ }_{\gamma \delta}$ in (4.1) it is easy to derive the possible Petrov types of the field, which depend on the different values of the constant $A$. The three eigenvalues $\lambda_{i}$ of the Riemann tensor are

$$
\begin{aligned}
& \lambda_{1}=-\frac{4\left(1-A^{2}\right)}{\left(A^{2}+3\right)^{2}} \frac{1}{R^{2}} \\
& \lambda_{2}=+\frac{2\left(1-A^{2}\right)(1-A)}{\left(A^{2}+3\right)^{2}} \frac{1}{R^{2}} \\
& \lambda_{3}=+\frac{2\left(1-A^{2}\right)(1+A)}{\left(A^{2}+3\right)^{2}} \frac{1}{R^{2}} .
\end{aligned}
$$

Under the condition $\lambda_{i}$ to be finite in the interior case (i.e. $R=0$ included) $A$ must be \pm 1 or $\pm \infty$ (not only $A=+1$, as Davies and Caplan postulated), and hence $\lambda_{i}=0$ (Petrov type 0), i.e. spacetime is (at least locally) flat.

In the exterior case ( $R=0$ excluded) $\lambda_{i}$ are finite for all possible values of $A$.

\section{a) General Case $A$}

The three eigenvalues are different from each other and the Petrov type is the general case $I$, or more exactly $I_{5}$ in the notation of Jordan, Ehlers and Kundt [5].

$$
\text { b) } A=0, A= \pm 3
$$

In this case degenerate type $D$. 


$$
\text { c) } A= \pm 1, A= \pm \infty
$$

The three eigenvalues vanish, i.e. Petrov type 0 , spacetime is (locally) flat.

Wemention, that the type is the same over the whole region $R_{1} \leqq R \leqq R_{2}$, if the region is not separated by matter.

\section{Conclusion}

It is obvious, that one should try to fit the interior solution to the outside field. Indeed, in case the matter distribution, which generates the field, has the structure of a surface density (infinitely thin hollow cylinder), the fitting procedure is not too complicated. A paper, which discusses this procedure, the physical properties of the hollow cylinder and the generated field in full detail, is in preparation.

I thank Dr. W. Graf for drawing my attention to the Kasner solution.

Work supported by the Deutsche Forschungsgemeinschaft.

\section{References}

1. Levy,H., Robinson, W.J.: The rotating body problem. Proc. Camb. Phil. Soc., 60, 279-285 (1964).

2. Davies, H., Caplan,T.A.: The space-time metric inside a rotating cylinder. Proc. Camb. Phil. Soc., 69, 325-327 (1971).

3. Kasner, E.: Trans. Amer. Math. Soc. 27, 249 (1925).

4. Landau,L.D., Lifschitz,E.M.: Klassische Feldtheorie. Berlin: Akademie-Verlag 1967.

5. Jordan, P., Ehlers,J., Kundt, W.: Strenge Lösungen der Feldgleichungen der Allgemeinen Relativitätstheorie. Akad. Wiss. Lit. (Mainz), Abhand. Math.-Nat. Kl.1, 1961.

Eckart Frehland

Physikalisches Institut d. Universität

Abt. Theoretische Physik

BRD-7800 Freiburg

Hermann-Herder-Str. 3 